\title{
A weighted logarithmic barrier interior-point method for linearly constrained optimization
}

\author{
Selma Lamri, Bachir Merikhi and Mohamed Achache
}

\begin{abstract}
In this paper, a weighted logarithmic barrier interior-point method for solving the linearly convex constrained optimization problems is presented. Unlike the classical central-path, the barrier parameter associated with the perturbed barrier problems, is not a scalar but is a weighted positive vector. This modification gives a theoretical flexibility on its convergence and its numerical performance. In addition, this method is of a Newton descent direction and the computation of the step-size along this direction is based on a new efficient technique called the tangent method. The practical efficiency of our approach is shown by giving some numerical results.
\end{abstract}

Mathematics Subject Classification (2010): 90C30, 90C25, 90C51.

Keywords: Logarithmic barrier method, linearly constrained convex optimization, interior-point methods.

\section{Introduction} problem:

In this paper, we consider the linearly convex constrained optimization (LCCO)

$$
\bar{p}=\min f(x) \text { subject to } x \in \mathcal{F},
$$

where the objective function $f: \mathbb{R}^{n} \rightarrow \mathbb{R}$ is twice differentiable and convex over the feasible set $\mathcal{F}=\left\{x \in \mathbb{R}^{n}: x \geq 0, A x=b\right\}, A$ is a given $(m \times n)$ matrix with full rank row $m$ and $b \in \mathbb{R}^{m}$.

This problem has many important applications in theory as well in practice. In particular, it includes linear and quadratic optimization. Feasible logarithmic barrier interior-point methods gained much more attention than others. Their derived algorithms enjoy some interesting results such as polynomial complexity and numerical efficiency. However, these algorithms require that the starting point must be strictly feasible and close to the central-path.This is a hard practical task to release and even impossible. On the other hand, at each iteration, they compute a descent direction and 
determine a step-size on this direction. It is known that computing this latter is very expensive while using classical line search methods. In order to overcome these two difficulties, we suggest for the first, a weighted-path (see [1], [2], [3], [8], [10], [12]) where a relaxation parameter associated with perturbed problems is introduced in order to give more flexibility on the numerical aspects. Beside, we propose a new numerical efficient procedure called the tangent method for determining this displacement. Across these two modifications the numerical results obtained by our algorithm are totally improved with respect to the classical logarithmic barrier interior-point approach (see [10], [13]). The paper is organized as follows. In section 2, perturbed relaxation problems based on the weighted barrier penalization are given where the convergence to the original problem is studied. The computation of the direction and of the step-size are stated. Finally, a weighted-path interior-point algorithm is presented. In section 3 , some numerical results are given to show the efficiency of our approach. Finally a conclusion and remarks end the section 4 .

\section{The weighted barrier penalization}

Throughout the paper, we assume that the following assumptions hold.

1. There exit a strictly feasible point $x_{0}>0$ such that $A x_{0}=b$.

2. The set of optimal solutions of $P$ is non empty bounded set.

It follows from the second hypothesis that

$$
\left\{d \in \mathbb{R}^{n}: f_{\infty}(d) \leq 0, d \geq 0, A d=0\right\}=\{0\},
$$

where $f_{\infty}$ denote the recession function of $f$. We deduce from the optimality conditions that $x^{*}$ is a solution of $P$ if and only if there exists an $y^{*} \in \mathbb{R}^{m}$ and $z^{*} \in \mathbb{R}^{n}$ such that

$$
\nabla f\left(x^{*}\right)+A^{T} y^{*}=z^{*} \geq 0, \quad A x^{*}=b, \quad\left\langle z^{*}, x^{*}\right\rangle=0, x^{*} \geq 0 .
$$

\subsection{The weighted perturbed problems}

Let us define the function $\theta: \mathbb{R} \times \mathbb{R} \rightarrow(-\infty,+\infty]$ by

$$
\theta(t, w)=\left\{\begin{array}{lcc}
t(\log t-\log w) & \text { if } & t>0, w>0, \\
0 & \text { if } & t=0, w \geq 0, \\
+\infty & \text { otherwise. } &
\end{array}\right.
$$

The function $\theta$ is convex, lower semi-continuous and proper. We consider now the following function defined on $\mathbb{R}_{+}^{n} \times \mathbb{R}_{+}^{n}$ by

$$
\varphi(\mu r, x)= \begin{cases}f(x)+\sum_{i=1}^{n} \theta\left(\mu r_{i}, x_{i}\right) & \text { if } x \in \mathcal{F}, \\ +\infty & \text { otherwise }\end{cases}
$$

where $\mu>0$ is the barrier parameter and $r=\left(r_{1}, r_{2}, \ldots, r_{n}\right)^{T} \in \mathbb{R}_{+}^{n}$, the vector of the weight associated with the barrier function.

Finally, we introduce the function $p^{r}$ defined by

$$
p^{r}(\mu)=\inf _{x}\left[\varphi_{\mu}^{r}(x)=\varphi(\mu r, x): x \in \mathbb{R}^{n}\right] .
$$


The function $p^{r}$ is convex since $\varphi_{\mu}^{r}$ is convex. By construction, $P_{0}^{r}$ is only the problem $P$ with $\bar{p}=p^{r}(0)$. The function $\varphi_{\mu}^{r}$ is convex, lower semi-continuous and proper, its recession function is given by

$$
\left(\varphi_{\mu}^{r}\right)_{\infty}(d)=\lim _{\alpha \rightarrow+\infty} \frac{\varphi_{\mu}^{r}\left(x_{0}+\alpha d\right)-\varphi_{\mu}^{r}\left(x_{0}\right)}{\alpha} .
$$

We obtain

$$
\left(\varphi_{\mu}^{r}\right)_{\infty}(d)=\left\{\begin{array}{ll}
f_{\infty}(d) & \text { if } \\
+\infty & \text { otherwise. }
\end{array} \quad d \geq 0, A d=0\right.
$$

Then

$$
\left\{d \in \mathbb{R}^{n}:\left(\varphi_{\mu}^{r}\right)_{\infty}(d) \leq 0\right\}=\left\{d \in \mathbb{R}^{n}: f_{\infty}(d) \leq 0, d \geq 0, A d=0\right\},
$$

where $d$ is the descent direction and $\alpha$ is the step-size.

Since this set is reduced to $\{0\}$ then the problem $P_{\mu}^{r}$ admits an optimal solution for each $\mu>0$. The function $\varphi_{\mu}^{r}$ is strictly convex for all $\mu \geq 0$ and $r \geq 0$, then $P_{\mu}^{r}$ has an unique optimal solution denoted by $x_{\mu}^{r}$.

\subsection{Convergence of the weighted perturbed solutions to the optimal solution of $P_{\mu}^{r}$}

The necessary and sufficient optimality conditions of $\left(P_{\mu}^{r}\right)$ imply that there exits $y_{\mu}^{r}=y(\mu, r) \in \mathbb{R}^{m}$, such that

$$
\begin{aligned}
\nabla f\left(x_{\mu}^{r}\right)-\mu X^{-1} r+A^{T} y_{\mu}^{r} & =0, \\
A x_{\mu}^{r} & =b,
\end{aligned}
$$

where $X=\operatorname{Diag}\left(x_{\mu}^{r}\right)$.

Note that $y_{\mu}^{r}$ is uniquely defined since $A$ is of full rank row. In fact, the couple $\left(x_{\mu}^{r}, y_{\mu}^{r}\right)$ is the solution of the system $H(x, y)=0$ where

$$
H(x, y)=\left(\begin{array}{l}
\nabla f(x)-\mu X^{-1} r+A^{T} y \\
A x-b
\end{array}\right) .
$$

By the implicit function theorem, the functions $\mu \mapsto x(\mu, r)=x_{\mu}^{r}$ and $\mu \mapsto y(\mu, r)=$ $y_{\mu}^{r}$ are differentiable on $(0, \infty)$ and we have,

$$
\left(\begin{array}{cc}
\nabla^{2} f\left(x_{\mu}^{r}\right)+\mu R X^{-2} & A^{T} \\
A & 0
\end{array}\right)\left(\begin{array}{c}
x^{\prime}(\mu, r) \\
y^{\prime}(\mu, r)
\end{array}\right)=\left(\begin{array}{c}
X^{-1} r \\
0
\end{array}\right)
$$

where $R=\operatorname{Diag}(r)$, it follows that the function $p^{r}$ is differentiable on $(0, \infty)$. Recall that

$$
p^{r}(\mu)=f\left(x_{\mu}^{r}\right)+\mu \sum_{i=1}^{n} r_{i}\left(\ln \mu r_{i}-\ln \left(x_{i}\right)_{\mu}^{r}\right),
$$

and then

$$
\left(p^{r}(\mu)\right)^{\prime}=\sum_{i=1}^{n} r_{i}\left(1+\ln \mu r_{i}-\ln \left(x_{i}\right)_{\mu}^{r}\right)+\left\langle\nabla f\left(x_{\mu}^{r}\right)-\mu X^{-1} r, x^{\prime}(\mu, r)\right\rangle .
$$


In view of (2.2) and (2.4)

$$
\begin{aligned}
\left(p^{r}(\mu)\right)^{\prime} & =\sum_{i=1}^{n} r_{i}\left(1+\ln \mu r_{i}-\ln \left(x_{i}\right)_{\mu}^{r}\right)-\left\langle A^{T} y_{\mu}^{r}, x^{\prime}(\mu, r)\right\rangle \\
& =\sum_{i=1}^{n} r_{i}\left(1+\ln \mu r_{i}-\ln \left(x_{i}\right)_{\mu}^{r}\right)-\left\langle y_{\mu}^{r}, A x^{\prime}(\mu, r)\right\rangle, \\
& =\sum_{i=1}^{n} r_{i}\left(1+\ln \mu r_{i}-\ln \left(x_{i}\right)_{\mu}^{r}\right) .
\end{aligned}
$$

Since $x_{\mu}^{r} \in \mathcal{F}$ and $p^{r}$ is convex we obtain:

$$
f\left(x_{\mu}^{r}\right) \geq \bar{p}=p^{r}(0) \geq p^{r}(\mu)+(0-\mu)\left(p^{r}(\mu)\right)^{\prime}=f\left(x_{\mu}^{r}\right)-\mu\|r\|_{1} .
$$

Consequently, we have

$$
\bar{p} \leq f\left(x_{\mu}^{r}\right) \leq \bar{p}+\mu\|r\|_{1}
$$

Then if

$$
\mu \mapsto 0, f\left(x_{\mu}^{r}\right)=\bar{p} .
$$

Now, we interested to the weighted-path of $\left\{x_{\mu}^{r}\right\}$ when $\mu \mapsto 0$.

i) Case where $f$ is strongly convex with coefficient $\tau>0$. Hence $P$ has a unique optimal solution $x^{*}$, and we have

$$
\mu\|r\|_{1} \geq f\left(x_{\mu}^{r}\right)-f\left(x^{*}\right) \geq\left\langle\nabla f\left(x^{*}\right), x_{\mu}^{r}-x^{*}\right\rangle+\frac{\tau}{2}\left\|x_{\mu}^{r}-x^{*}\right\|^{2} .
$$

In view of (2.1), we deduce

$$
\begin{gathered}
\mu\|r\|_{1} \geq\left\langle z^{*}, x_{\mu}^{r}\right\rangle+\frac{\tau}{2}\left\|x_{\mu}^{r}-x^{*}\right\|^{2} \geq \frac{\tau}{2}\left\|x_{\mu}^{r}-x^{*}\right\|^{2}, \\
\left\|x_{\mu}^{r}-x^{*}\right\| \leq \sqrt{\frac{2 \mu\|r\|_{1}}{\tau}} .
\end{gathered}
$$

ii) For the case where $f$ is only convex is more complex. Note first that for $\mu \leq 1$,

$$
x_{\mu}^{r} \in\left\{x: x \geq 0, A x=b, f(x) \leq\|r\|_{1}+\bar{p}\right\} .
$$

This set is closed convex non empty. Its recession cone is

$$
\left\{d \in \mathbb{R}^{n}: f_{\infty}(d) \leq 0, d \geq 0, A d=0\right\}=\{0\} .
$$

By the second assumption the set of optimal solutions of $P$ is bounded which implies that each adherence value of $\left\{x_{\mu}^{r}\right\}$ when $\mu \mapsto 0$ is an optimal solution of $P$.

Remark 2.1. If $r=e$, where $e$ is the vector of ones, then the weighted-path coincides with the classical path (see[6]). 


\subsection{The description of the method}

Letting $\mathcal{F}^{0}=\left\{x \in \mathbb{R}^{n}: x>0, A x=b\right\}$ the set of strictly feasible points. The principle of the method is as follows: Let $\left(\mu_{k} r, x_{k}\right) \in \mathbb{R}_{+}^{n} \times \mathcal{F}^{0}$, the current iterate.

1. We make an approximated minimization of the weighted perturbed $P_{\mu_{k}}^{r}$ which gives a new point $x_{k+1}$ such that $\varphi\left(\mu_{k+1} r, x_{k+1}\right)<\varphi\left(\mu_{k} r, x_{k}\right)$.

2. We take $\mu_{k+1}<\mu_{k}$.

We iterated until we obtained an approximated optimal solution of the original problem. The weighted perturbed problem is defined by

$$
\min _{x} \varphi_{\mu}^{r}(x)=\min _{x}\left[f(x)+\sum_{i=1}^{n} \theta\left(\mu r_{i}, x_{i}\right): x \in \mathcal{F}\right] .
$$

\subsection{The Newton descent direction}

At $x \in \mathcal{F}^{0}$, the Newton descent direction $d$ is given by solving the following quadratic convex program:

$$
\min _{d}\left[\left\langle\nabla \varphi_{\mu}^{r}(x), d\right\rangle+\frac{1}{2}\left\langle\nabla^{2} \varphi_{\mu}^{r}(x) d, d\right\rangle: A d=0\right] .
$$

It suffices to solve the linear system with $n+m$ equations

$$
\left(\begin{array}{cc}
\nabla^{2} f(x)+\mu R X^{-2} & A^{T} \\
A & 0
\end{array}\right)\left(\begin{array}{c}
d \\
s
\end{array}\right)=\left(\begin{array}{c}
\mu X^{-1} r-\nabla f(x) \\
0
\end{array}\right),
$$

where $s \in \mathbb{R}^{m}$.

It easy to prove that the linear system (2.5) has a unique solution. The descent direction being thus obtained, it is now question of minimizing a function of one real variable to obtain the step-size $\alpha$.

$$
\gamma^{r}(\alpha)=\varphi_{\mu}^{r}(x+\alpha d)-\varphi_{\mu}^{r}(x)=f(x+\alpha d)-f(x)-\mu \sum_{i=1}^{n} r_{i} \ln \left(1+\alpha t_{i}\right),
$$

where $t=X^{-1} d$, the function $\gamma^{r}$ is convex.

Next task, we propose a new method to determine the step-size.

\subsection{A tangent method for determining the step-size}

Our approach is try out a sequence of candidate values for $\alpha$, when the condition $\left(\gamma^{r}(\alpha)\right)^{\prime} \leq \epsilon$ is satisfied, stopping and accept this value. We can say that this technique is done in two phases.

1. The first phase finds an interval containing the required step-size, the choice of the bounds of the interval is similar to the bisection method, when we restrict the value of $\alpha$ until we find the required value.

2. The second phase computes the optimal step-size within this interval, in this phase we determine the tangents $T_{1}$ and $T_{2}$ in the bounds of the interval and we select the value corresponding to the intersection of the tangents $T_{1}$ and $T_{2}$. 
The upper bound on the step-size $\alpha$ is given by

$$
\alpha_{\max }=\min \left\{-\frac{x_{i}}{d_{i}} ; \quad i \in \hat{I}\right\},
$$

where

$$
\hat{I}=\left\{i: \quad d_{i}<0\right\}
$$

Because the convexity of the function $\gamma^{r}(\alpha)$, this technique will be more efficiency in practice, the next figures shows clearly this idea:

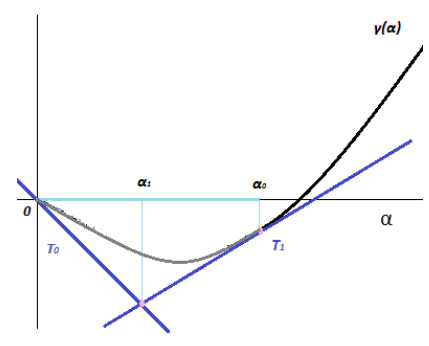

Figure 1

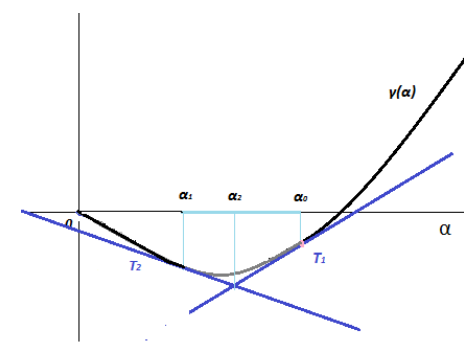

Figure 2

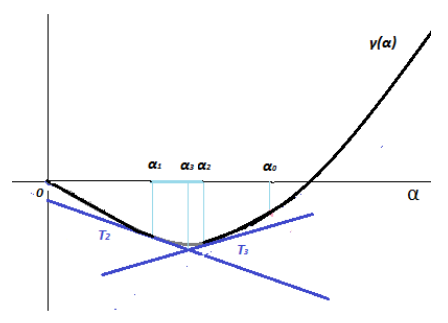

Figure 3 
The tangent algorithm for determining the step-size is follows.

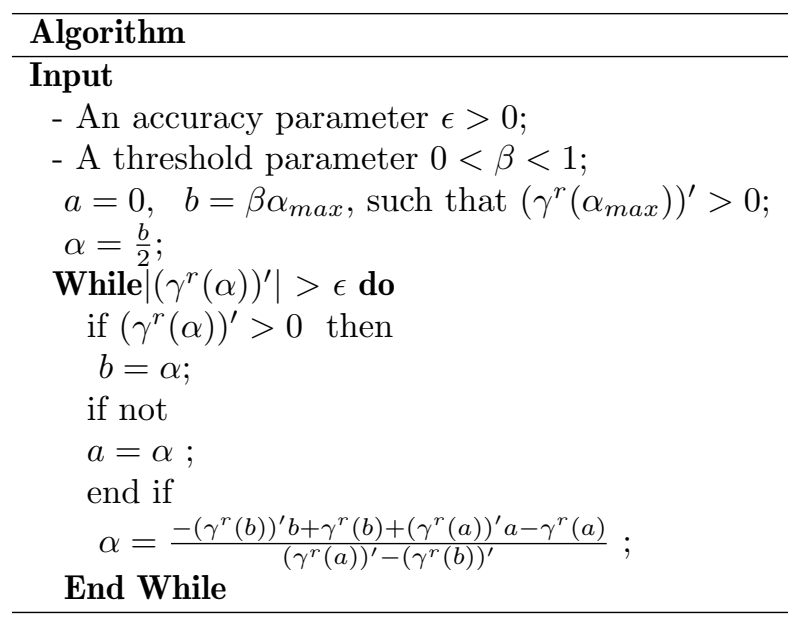

We are now ready to state the generic algorithm for solving LCCO.

\section{The generic algorithm}

Threshold parameters $\epsilon>0, \bar{\mu}>0$ and $\lambda \in[0,1]$, are given;

Start with $x_{0} \in \mathcal{F}^{0}, \mu>\bar{\mu}$ and a weight vector $r>0$;

1) Solve the linear system (2.5) to obtain $d$;

2) Take $t=X^{-1} d$;

If $\|t\| \geq \epsilon$

- Determinate $\tilde{\alpha}$ with tangent method ;

- Update $x_{k+1}=x_{k}+\tilde{\alpha} d_{k}, \mu_{k+1}=\lambda \mu_{k}$ and return to 1 ;

If $\|t\| \leq \epsilon$

Case 1. $\mu_{k} \leq \bar{\mu}$

STOP we have obtained a good approximation of the optimal solution of $P$;

Case 2. $\mu_{k}>\bar{\mu}$

We have obtained a good approximation of $p^{r}(\mu)$, do $\mu_{k+1}=\lambda \mu_{k}$ and go to 1 ;

\section{Numerical results}

In the following section, we apply our algorithm on some different examples of LCCO. A comparative numerical tests with a classical line search are presented, Our implementation is done by the Scilab 5.4.1. We use in the sequel the following notation.

Method 1: the first alternative uses the tangent technique.

Method 2: the second alternative uses the Wolfe method. 
Outer: the number of outer iterations.

Inner: the number of inner iterations.

Objective: the optimal value of the objective function $\bar{p}$.

Time: the time measured in seconds. Our tolerance is $\epsilon=10^{-6}$ in all our testing examples.

The below examples are taken from literature [10], the numerical obtained results with different values of $\mathrm{r}$ such as $r_{1}=(0.9,1,0.03)^{T}, r_{2}=(2,1,3,1,4)^{T}$, $r_{3}=(1,1,0.4,1,0.4,1,0.4,0.4,1,0.96)^{T}$ and

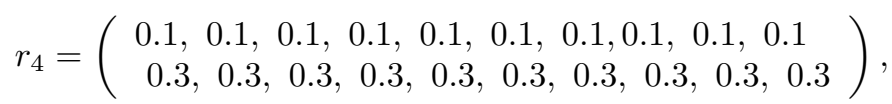

are summarized in table 1.

\begin{tabular}{|c|c|c|c|c|c|}
\hline \multirow[b]{2}{*}{ Example } & \multirow[b]{2}{*}{ Size $(m, n)$} & \multicolumn{2}{|c|}{ Method 1} & \multicolumn{2}{|c|}{ Method 2} \\
\hline & & Inner & Outer & Inner & Outer \\
\hline 1 & $(2,3)$ & 7 & 7 & 162 & 7 \\
\hline 2 & $(3,5)$ & 24 & 6 & 46 & 8 \\
\hline 3 & $(3,10)$ & 20 & 7 & 40 & 8 \\
\hline 4 & $(10,20)$ & 25 & 7 & 83 & 7 \\
\hline
\end{tabular}

Table 1.

In the following, we compare our approach with the classical path method (non weighted case).

Example with variable size. We consider the following LCCO problem:

$$
\bar{p}=\min [f(x): x \geq 0, A x=b],
$$

where $f(x)=\sum_{i=1}^{n} x_{i} \ln x_{i}, b_{i}=1$ and

$$
A[i, j]=\left\{\begin{array}{ll}
1 & \text { if } i=j \text { or } j=i+m), \\
0 & \text { else, }
\end{array}\right\} \text {, with } n=2 m .
$$

The strictly feasible starting point is:

$$
x^{0}=\left(\begin{array}{lllll}
0.7, & \ldots, & 0.7, & 0.3, \quad \ldots, & 0.3
\end{array}\right)^{T} .
$$

The exact solution is:

$$
x^{*}=(0.5, \quad 0.5, \quad \cdots \quad, 0.5)^{T} .
$$

The optimal values with different size of $n$ are:

\begin{tabular}{|l|l|l|l|}
\hline$n$ & 20 & 400 & 900 \\
\hline Objective & -6.9314718 & -138.62944 & -311.911623 \\
\hline
\end{tabular}

The obtained numerical results with different size of $n$ and barrier parameter $\mu$ are stated in tables 2 and 3 . 


\section{Weighted case}

\begin{tabular}{|c|c|c|c|c|c|c|}
\hline \multirow[b]{2}{*}{$\mu$} & \multicolumn{2}{|c|}{$n=20$} & \multicolumn{2}{|c|}{$n=400$} & \multicolumn{2}{|c|}{$n=900$} \\
\hline & Outer & Time & Outer & Time & Outer & Time \\
\hline 0.01 & 2 & $0.296 \times 10^{-3}$ & 2 & 0.17160 & 2 & 23.476212 \\
\hline 0.25 & 4 & $0.316 \times 10^{-3}$ & 4 & 0.28762 & 4 & 32.40515 \\
\hline 1 & 5 & $0.499 \times 10^{-3}$ & 5 & 0.355702 & 5 & 41.84014 \\
\hline 5 & 6 & $0.665 \times 10^{-3}$ & 6 & 0.400961 & 6 & 52.96016 \\
\hline
\end{tabular}

Table 2.

\section{Non weighted case}

\begin{tabular}{|c|c|c|c|c|c|c|}
\hline \multirow[b]{2}{*}{$\mu$} & \multicolumn{2}{|c|}{$n=20$} & \multicolumn{2}{|c|}{$n=400$} & \multicolumn{2}{|c|}{$n=900$} \\
\hline & Outer & Time & Outer & Time & Outer & Time \\
\hline 0.01 & 4 & $0.325 \times 10^{-3}$ & 4 & 0. 2915518 & 4 & 33.147112 \\
\hline 0.25 & 6 & $0.482 \times 10^{-3}$ & 6 & 0.3832547 & 6 & 40.40114 \\
\hline 1 & 7 & $0.591 \times 10^{-3}$ & 7 & 0.4512415 & 7 & 49.88745 \\
\hline 5 & 8 & $0.835 \times 10^{-3}$ & 8 & 0.6001489 & 8 & 58.91456 \\
\hline
\end{tabular}

\section{Table 3.}

\section{Conclusion and remarks}

In this paper we have introduced a relaxation of the classical path of the perturbed LCCO problem and we have presented a new technique for determining the step-size. These have a great influence on the acceleration of the convergence of the algorithm i.e., the number of iterations and the time produced are reduced significantly. This analysis may be extended to inducing a general weight vector $w>0$ as the barrier parameter instead of the form $\mu r$ with $r>0$.

\section{References}

[1] Achache, M., A weighted-path-following method for the linear complementarity problem, Stud. Univ. Babeş-Bolyai Informatica, 49(2004), no. 1, 61-73.

[2] Achache, M., A polynomial-time weighted path-following interior-point algorithm for linear optimization, Asian-Eur. J. Math., 13(2020), no. 1, 1-9.

[3] Achache, M., Khebchache, R., A full-Newton step fessible weighted primal-dual interior point algorithm for monotone LCP, Afr. Mat., 27(2016), no. 3, 591-601.

[4] Achache, M., Khebchache, R., A full-Newton step feasible weighted primal-dual interior point algorithm for monotone LCP, Afr. Mat., 27(2016), no. 3, 591-601.

[5] Alzalg, B., A logarithmic barrier interior-point method based on majorant functions for second-order cone programming, Department of Mathematics, The University of Jordan, Amman 11942, Jordan, (2017). 
[6] Bachir Cherif, B., Merikhi, B., A penalty method for nonlinear programming, RAIRO Oper. Res., 53(2019), no. 1, 29-38.

[7] Crouzeix, J.-P., Seeger, A., New bounds for the extreme values of a finite sample of real numbers, J. Math. Anal. Appl., 197(1996), 411-426.

[8] Crouzeix, J.-P., Merikhi, B., A logarithm barrier method for semi-definite programming, RAIRO Oper. Res., 42(2008), no. 2, 123-139.

[9] Darvay, Zs., A weighted-path-following method for linear optimization, Stud. Univ. Babeş Bolyai Informatica, 47(2002), no. 1, 3-12.

[10] Goutali, M., Complexité et implimentation numérique d'une méthode de points interieurs pour la programmation convexe, Mémoire de Doctorat, Dept. Math. Univ. Sétif, 2018.

[11] Kebbiche, Z., Benterki,D., A weighted-path-following method for linearly constrained convex programming, Rev. Roumaine Math. Pures Appl., 57(2012), no. 3, 245-256.

[12] Kettab, S., Benterki, D., A relaxed logarithmic barrier method for semidefinite programming, RAIRO Oper. Res., 49(2015), 555-568.

[13] Menniche, L., Benterki, D., Alogarithmic barrier approach for linear programming, J. Comput. Appl. Math., 312(2017), 267-275.

[14] Zhang, M., Bai, Y., Wang, G., A new primal-dual path-following interior-point algorithm for linearly constrained convex optimization, J. Shanghai Univ., 12(2008), no. 6, 475-480.

Selma Lamri

Laboratoire de Mathématiques Fondamentales et Numériques,

Sétif1, Sétif 19000, Algérie

e-mail: selmalamri@yahoo.com

Bachir Merikhi

Laboratoire de Mathématiques Fondamentales et Numériques,

Sétif1, Sétif 19000, Algérie

e-mail: bmerikhi@univ-setif.dz

Mohamed Achache

Laboratoire de Mathématiques Fondamentales et Numériques,

Sétif1, Sétif 19000, Algérie

e-mail: achache_m@univ-setif.dz 